\title{
Drug-resistant tuberculosis can be predicted by Mycobacterial interspersed repetitive unit locus
}

\author{
Wen Yu-feng ${ }^{1 *}{ }^{*}$, Jiang Chao ${ }^{1 \dagger}$ and Cheng Xian-feng ${ }^{2}$ \\ ${ }^{1}$ School of Public Health, Wannan Medical College, Wuhu, China \\ 2 Institute of Dermatology, Chinese Academy of Medical Sciences and Peking Union, Nanjing, China
}

\section{Edited by:}

Henk Aarts, National Institute for

Public Health and the Environment,

Netherlands

\section{Reviewed by:}

Noton Kumar Dutta, Johns Hopkins

University, USA

Giovanna Riccardi, University of

Pavia, Italy

\section{*Correspondence:}

Wen Yu-feng, School of Public

Health, Wannan Medical College,

Wen Chang road No. 22, Wuhu

241002, China

e-mail:wyf@wnmc.edu.cn

these authors have contributed equally to this work.
It is unknown whether MIRU-VNTR (Mycobacterial Interspersed Repetitive Unit-Variable Number of Tandem Repeat) is associated with drug resistance of Mycobacterium tuberculosis. The purpose of this study was to explore the ability of $24 \mathrm{MIRU}$ loci to predict the drug resistance of Isoniazid (INH), Rifampicin (RFP), Streptomycin (SM), Ethambutol (EMB) and Pyrazinamide (PZA). We collected the drug resistance and MIRU loci information of 109 strains of $M$. tuberculosis from an open database. The results of multivariate logistic regression showed that the VNTR polymorphism of MTUB04 was related to INH resistance [odds ratio $(O R)=2.82, P=0.00$ ], RFP resistance $(O R=1.91$, $P=0.02), S M$ resistance $(O R=1.98, P=0.01)$ and $E M B$ resistance $(O R=1.95$, $P=0.03)$. MIRU40 was associated with INH resistance $(O R=2.22, P=0.00)$. MTUB21 was connected with $I \mathrm{NH}$ resistance $(O R=1.63, P=0.02)$ and $S M$ resistance $(O R=1.69$, $P=0.01)$. MIRU26 was correlated with $S M$ resistance $(O R=1.52, P=0.04)$. MIRU39 was associated with $\mathrm{EMB}$ resistance $(O R=4.07, P=0.02)$. The prediction power of MIRU loci were $0.84,0.70,0.85$, and 0.74 respectively for INH (predicted by MTUB04, MIRU20, and MTUB21), RFP (predicted by MTUB04), SM (predicted by MTUB21 and MIRU26) and EMB (MTUB04 and MIRU39) through ROC analysis. Our results showed that MIRU loci were related to anti-tuberculosis drug and could predict the drug resistance of tuberculosis.

Keywords: tuberculosis, drug resistance, variable number of tandem repeats, MIRU, Mycobacterial interspersed repetitive unit

\section{INTRODUCTION}

According to the Global Tuberculosis Report 2013, 8.6 million people were infected with tuberculosis (TB), and 1.3 million people died from TB in 2012, and there were $3.6 \%$ of new cases and $20.0 \%$ of retreatment were infected with Multidrug-resistant TB $(\text { MDR-TB })^{1}$. Environment (Lalor et al., 2013), including nutritional status, inadequacy of treatment and gene mutation were the main risk factors of drug-resistant tuberculosis. Among genetic factors, the efflux pump (Da Silva et al., 2011) and gene mutation (Nebenzahl-Guimaraes et al., 2014) have been widely used to explain the formation of anti-tuberculosis drug resistance. A recent study found that there was a correlation between genotypes of tuberculosis strains and drug-resistant tuberculosis (Liu et al., 2012). Mycobacterial interspersed repetitive unit-variable number of tandem repeat (MIRU-VNTR) method is widely used in genotyping the tuberculosis strains. The essence of MIRU (a total of 41, (Supply et al., 2000) in the tuberculosis) was a variable number tandem repeat, of which the number were highly polymorphic.

The exact biological mechanisms underlying the effects of VNTR on mycobacteria remain unclear. Some researchers argued that (Supply et al., 1997) it may play a role in the regulation

\footnotetext{
${ }^{1}$ http://www.who.int/tb/publications/global_report/en/
}

of gene expression, e.g., the operon of the polycistron having differential gene translation and the structure of the chromosome. Akhtar et al. (2009) reported that when the number of repeat unit of MTUB39 was 4, the expression of the downstream gene $l p d A$ was 12 times greater than when the number of was 1 . Similar result was also reported in another experiment (Perez-Lago et al., 2013), which found a 1.56-fold difference in $l p d A$ when the number of repeat unit of MTUB39 was 4 instead of 3 . This research also found that there was no difference in the expression of downstream gene whenever the number of repetition of MIRU10 was 4 or 3. Tantivitayakul et al. (2010) found that there was a lower expression of downstream gene $g f p$ when the number of repeat unit of MIRU10 was from 4 to 7 copies. In addition, different tandem repeats may affect the folding of DNA, thereby affecting the attraction, binding and interaction (Olsen et al., 2009) of transcription factor. Existing evidence strongly indicated that MIRU locus may exert a regulation function on the expression of genes nearby. Nowadays MIRU-VNTR method is widely used to analyze the genotyping, epidemic information and other areas of tuberculosis strains. However, it is not yet clear whether MIRU locus is involved in the formation of drug-resistant tuberculosis or not, and its ability to predict the occurrence of drug-resistant tuberculosis is not well unveiled. Hence, in order to find a new way to predict the drug-resistant tuberculosis, we conducted a randomized case-control study (the data was from a published 
database) to explore the relationship between the MIRU locus and drug-resistant tuberculosis.

\section{SYSTEM AND METHODS}

109 strains were included in the study, of which 54 strains were from Germany, 20 strains from Ghana, 20 strains from Uganda and 15 strains from former Soviet Union. The inclusion criteria were expressed as follows: First, strains must be Mycobacterium tuberculosis. Second, MIRU-VNTR genotyping results of the strains included 24 loci information. Third, there were drug resistance information about Isoniazid (INH), Rifampicin (RFP), Streptomycin(SM), and Ethambutol(EMB). Mycobacterium bovis, Mycobacterium africanum and other nontuberculosis mycobacteria were not included in this study. Strains of which the susceptibility test was positive were enrolled in the case group, while the sensitive strains were included in the control group.

24 loci and drug resistance data were derived from an online reference database of MIRU-VNTRplus website (http:// www.miru-vntrplus.org) (Allix-Beguec et al., 2008). The data in the reference database were provided by the Pasteur Institute in France and the German National Reference Center for Mycobacteria. Technical methods for detecting MIRU loci repetition could be referred to the technical manual supplied by the Pasteur Institute 2 .

Allelic diversity $h$ was used to measure the MIRU locus polymorphism. Univariate and non-conditional multivariate Logistic regression analysis were applied in MIRU locus prediction model. The dependent variable was drug resistance result, 1 standing for resistance, 0 standing for sensitivity. Independent variable was the number of repetitions of each MIRU locus (continuous variables). The ROC analysis was used to evaluate the effect of MIRU locus model which may predict drug-resistant tuberculosis. Maximum Youden Index was used to determine the predictor of model. Functional genes near the MIRU locus were analyzed by artemis software. According to the upstream and downstream primers of the study locus, the position of MIRU locus in the genome of standard strains of $M$. tuberculosis H37Rv was determined. Moreover, the functions of the upstream and downstream genes of MIRU loci were analyzed.

\section{RESULTS \\ DISTRIBUTION OF THE VNTR, POLYMORPHISM AND DRUG RESISTANCE OF MIRU LOCI}

The number of the repeat unit of 24 MIRU loci ranged from 0 to 13. The minimum $h$ value of MIRU27 was 0.10 while QUB 26 was 0.81 . Drug resistance rate that corresponded to different copies of each MIRU locus ranged from 0 to $100 \%$ (Table in Supplementary information).

\section{UNIVARIATE ANALYSIS OF FACTORS OF ANTI-TUBERCULOSIS DRUG RESISTANCE}

Through the univariate logistic regression analysis revealed a significant relationship between loci and anti-tuberculosis drug

${ }^{2}$ http://www.miru-vntrplus.org/MIRU/files/MIRU-VNTRtypingmanualv6. pdf resistance. Specifically, for INH resistance, there were MTUB04, MIRU40, MIRU16, MTUB21, MTUB21, MTUB30, MIRU26, MIRU31, MTUB39, OUB26, and MIRU39. For RFP resistance, two loci were MTUB04 and OUB26. For SM resistance, there were MTUB04, MIRU16, MTUB21, MIRU26, MIRU31, MTUB39, OUB26, MIRU39. For EMB resistance, there were MTUB04, OUB26 and MIRU39 (Table in Supplementary information).

\section{MULTIVARIATE ANALYSIS OF FACTORS OF ANTI-TUBERCULOSIS DRUG RESISTANCE}

Loci which were statistically significant in Table 2 were set up as the independent variables. The multivariate logistic regression suggested that loci which were significantly associated with antituberculosis drug resistance were as follows: for INH resistance model, there were MTUB04, MIRU40, and MTUB21; for RFP resistance model, there was MTUB04; for SM resistance model, there were MTUB04, MTUB21, and MIRU26; for EMB resistance model there were MTUB04 and MIRU39. In conclusion, there were 5 risk MIRU locus of drug resistance (Table $\mathbf{1}$ ).

\section{ROC ANALYSIS OF FOUR DRUG RESISTANCE}

The areas under the ROC curve (AUC) of INH, RFP, SM, and EMB were $0.84,0.70,0.85$, and 0.75 . The predictive values of prediction models of drug resistance ranged from 0.09 to 0.26 , and the Youden Index ranged from 0.40 to 0.61 (Table 2).

\section{BIOINFORMATICS ANALYSIS OF EACH MIRU LOCUS}

We search each MIRU locus and relative positions of functional genes adjacent to five MIRU loci from the genbank databases, founding that MTUB04 adjacent to $h s p R$ gene, MTUB21 adjacent to Rv1729c gene, MIRU40 adjacent to $r p l B$ gene, MIRU26 $R v 2680$ and MIRU39 adjacent to $e c c C b 1$ gene. The detailed information of each MIRU locus and relative positions of functional genes adjacent to five MIRU loci were listed (Table 3 ).

\section{DISCUSSION}

Our study showed that anti-tuberculosis drug resistance (INH, RFP, SM, and EMB) were well correlated with MTUB04, MTUB21, MIRU26, MIRU39, and MIRU40. It revealed that the drug resistance could be predicted by these loci. The prediction power of MIRU loci were $0.84,0.70,0.85$, and 0.74 respectively for INH (predicted by MTUB04, MIRU20, and MTUB21), RFP (predicted by MTUB04), SM (predicted by MTUB21 and MIRU26) and EMB (MTUB04 and MIRU39) through ROC analysis. Except for the poor sensitivity of resistance prediction model for EMB, the accuracy and sensitivity for other four models were high. Especially, MTUB04 locus had the ability to predict all four kinds of drug resistance, and MTUB21 locus could predict both INH and SM resistance.

Because report about the specific function of MIRU loci was not found, we suspected that some gene next to these loci may be paying an unknown role. For MTUB04, gene $h s p R$ was located at its upstream and there was $8 \mathrm{bp}$ overlapping between them. $\mathrm{HspR}$, binding to the HAIR (three HspR associated inverted repeats) sequence in the upstream of the $d n a K-h s p R$ operon, was a repressor of DnaK's (heat shock protein HSP70) (Narberhaus, 1999) expression, and the activity of HspR was regulated by the molecular chaperones DnaK (Bucca et al., 2000). Previous studies 
Table 1 | Multivariate analysis of influencing factors of four anti-tuberculosis drug resistances.

\begin{tabular}{|c|c|c|c|c|c|c|c|c|}
\hline Model & Loci & B & S.E. & Wald $\chi^{2}$ & $\mathbf{P}$ & OR & \multicolumn{2}{|c|}{ OR $95 \% \mathrm{Cl}$} \\
\hline \multirow{3}{*}{ INH } & MIRU40 & 0.80 & 0.24 & 10.81 & 0.00 & 2.22 & 1.38 & 3.58 \\
\hline & MTUB21 & 0.49 & 0.21 & 5.52 & 0.02 & 1.63 & 1.09 & 2.46 \\
\hline & Constant & -8.83 & 1.83 & 23.38 & 0.00 & 0.00 & & \\
\hline RFP & Constant & -3.97 & 0.94 & 17.94 & 0.00 & 0.02 & & \\
\hline \multirow[t]{3}{*}{ SM } & MTUB04 & 0.68 & 0.27 & 6.62 & 0.01 & 1.98 & 1.18 & 3.32 \\
\hline & MTUB21 & 0.53 & 0.20 & 6.63 & 0.01 & 1.69 & 1.13 & 2.52 \\
\hline & MIRU26 & 0.42 & 0.20 & 4.23 & 0.04 & 1.52 & 1.02 & 2.27 \\
\hline EMB & Constant & -7.64 & 1.95 & 15.42 & 0.00 & 0.00 & & \\
\hline
\end{tabular}

Table 2 | ROC analysis of four drug resistance.

\begin{tabular}{lcccccccc}
\hline Model & AUC & S.E. & P & AUC95\%Cl & $\begin{array}{c}\text { Positive if greater than } \\
\text { or equal toa }\end{array}$ & Sensitivity & 1-Specificity & Yueden index \\
\hline INH & 0.84 & 0.05 & 0.00 & $0.74-0.93$ & 0.24 & 0.83 & 0.22 \\
RFP & 0.70 & 0.09 & 0.02 & $0.54-0.87$ & 0.09 & 0.75 & 0.35 \\
SM & 0.85 & 0.04 & 0.00 & $0.77-0.94$ & 0.13 & 0.92 & 0.32 \\
EMB & 0.75 & 0.08 & 0.01 & $0.59-0.92$ & 0.26 & 0.54 & 0.40 \\
\hline
\end{tabular}

Table 3 | Bioinformatics analysis of relative gene adjacent to each MIRU locus.

\begin{tabular}{lcclc}
\hline Loci & Unit length & Repeat & Adjacent gene & Relative position \\
\hline MTUB04 & 51 & 2 & $h s p R$ & 8 bp duplication \\
MTUB21 & 33 & 2 & $R v 1729 c$ & $-13 \mathrm{bp}$ \\
MIRU40 & 54 & 1 & rp/B & $-46 \mathrm{bp}$ \\
MIRU26 & 51 & 3 & $R v 2680$ & $51 \mathrm{bp}$ duplication \\
MIRU39 & 47 & 2 & eccCb1 & $-3 \mathrm{bp}$ \\
\hline
\end{tabular}

showed that part sequence in the $3^{\prime}$ terminal of gene coding strand knock-out HspR almost had no binding activity with DnaK (Bandyopadhyay et al., 2012), then, causing the binding activity of HspR to HAIR reduced, and at last resulting in overexpression of DnaK. Xin et al. found (Xin et al., 2013) that in A549 cells, the overexpression of HSP70 could up-regulate the expression of Pgp transporter protein at transcriptional level. Meanwhile, INH, RFP, and EMB were the substrates of P-gp (Rakash et al., 2003; Hartkoorn et al., 2007; Pin-Fei, 2010), and the upregulation of Pgp could reduce drug concentration of INH, RFP, and EMB in mouse tissues (Rakash et al., 2003; Pin-Fei, 2010). Some studies have found that repeated sequences of $A L U$ could inhibit the expression of the reporter gene GFP in the upstream ( $\mathrm{Li}$ et al., 2012). Therefore, we hypothesized that increasing the number of repetitions unit of MTUB04 locus can inhibit the expression of HspR or the sequence of the $\mathrm{C}^{\prime}$ terminal of HspR, thus leading to the occurrence of drug resistance.

The gene $R v 1729 c$ in the downstream of MTUB21 locus, could express $\mathrm{S}$-adenosine methionine-dependent methyltransferases ${ }^{3}$. Strong evidences showed that the knockout of mmaA4 or hma encoding SAM-MTs proteins(Cole et al., 1998) may inhibit the synthesis of two kinds of Mycobacterium mycolic acid, Keto meromycolic and Oxygenate methyl meromycolic (Dubnau et al., 2000). Some studies showed that clinical efficacy of patients infected with isoniazid and rifampicin resistant M. tuberculosis strains could be improved with the use of delamanid inhibiting the synthesis of the two kinds of mycolic acid mentioned above (Gler et al., 2012). One of the mechanisms underlying for INH resistance is that INH oxidized by peroxidase attacks mycolic acid, the main constituent of the cell membrane of $M$. tuberculosis, resulting in the death of mycolic acid. The possible mechanism is that MTUB21 may exist as an enhancer to increase the transcription of $R v 1729 c$, resulting in the overexpression of mycolic acid, thus, forming the INH resistance. For SM resistance, the overexpression of mycolic acid may enhance the permeability barrier function of the cell wall, and then prevent penetration by SM.

\footnotetext{
${ }^{3} \mathrm{http} / / /$ www.ncbi.nlm.nih.gov/gene/885194
} 
The gene $R v 2680$ located in the downstream of MIRU26, the expression product of it may be the enoyl coenzyme A hydratase ${ }^{4}$. Enoyl-CoA hydratase is a key enzyme for the synthesis of M. tuberculosis meromycolic(Strong et al., 2003), involved in the oxidation of $\beta$ fatty acids. MIRU26 locus may serve as an enhancer to increase the overexpression of enoyl-CoA hydratase, and the penetration of the barrier function of the cell wall, thus resulting in the occurrence of SM resistance.

Existing studies found that when the number of repeat unit of MIRU39 locus was 4, the gene eccCb1 in the downstream had a higher transcription level (Refaya et al., 2012). The expression product of eccCb1 were ATP enzyme family members, constituting the EXS-1 transport channel together with Rv3870 for the transport of a variety of substrates (Champion et al., 2009) such as EspR (Rv3849), EspA (Rv3616c), EspB (Rv3881c), EspC (Rv3615c), ESAT-6, and CFP-10. We hypothesized that with the increasing number of the repeat unit of MIRU39 locus, the efficiency of the EXS-1 transport channel was increased, which then increased the efflux of EMB, and finally resulted in EMB resistance. However, whether EXS-1 transport channel has effect on the substrate EMB is still an open question.

Gene $r p l B$ which was in the downstream of MIRU40 locus may express $50 \mathrm{~S}$ ribosomal protein $\mathrm{L} 2^{5}$. Reports showed that it participated in the $50 \mathrm{~S}$ subunit's connection to $30 \mathrm{~S}$ ribosomal subunit, binded site of tRNA, contacting with16sRNA partly $^{6}$. However, the question that by what mechanisms can rplB allow M. tuberculosis to become resistant to INH still can not be explained.

Overall, our findings confirmed that some of the MIRU loci can predict the drug-resistant tuberculosis. The possible mechanism involved was that MIRU locus could increase or decrease the transcription of functional genes nearby, resulting in the upregulation of the expression of efflux pump, P-gp, meromycolic, mycolic acid and a plurality of other components. MIRU-VNTR was selected as the first choice for genotyping of M. tuberculosis by Centers for Disease Control and Prevention of U.S., due to simple operation and low cost (Cowan et al., 2012). Therefore, this study would not only enrich the knowledge of drug resistance of tuberculosis but also provide the probability of further exploration of the mechanism of anti-tuberculosis drug resistance. Meanwhile, it would be a potential cost-effective way to predict drug-resistant tuberculosis through MIRU loci method. However, the expression level of upstream and downstream genes of MIRU loci were not tested in our study and whether MIRU loci influence the expression of upstream and downstream gene through the mechanisms mentioned above still need further exploration.

\section{AUTHOR CONTRIBUTIONS}

This research was designed by WYF, written by JC, and the data was analyzed by CXF.

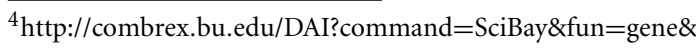
geneID $=887734$

${ }^{5}$ http://www.ncbi.nlm.nih.gov/gene/888341

${ }^{6} \mathrm{http}: / /$ www.uniprot.org/uniprot/I6Y4H2
}

\section{ACKNOWLEDGMENTS}

This publication made use of the MIRU-VNTRplus database website (http://www.miru-vntrplus.org/) developed by D. Harmsen, S. Nieman, P. Supply, and T. Weniger.

\section{SUPPLEMENTARY MATERIAL}

The Supplementary Material for this article can be found online at: http://www.frontiersin.org/journal/10.3389/fmicb. 2015.00147/abstract

\section{REFERENCES}

Akhtar, P., Singh, S., Bifani, P., Kaur, S., Srivastava, B. S., and Srivastava, R. (2009) Variable-number tandem repeat 3690 polymorphism in Indian clinical isolates of Mycobacterium tuberculosis and its influence on transcription. J. Med. Microbiol. 58, 798-805. doi: 10.1099/jmm.0.002550-0

Allix-Beguec, C., Harmsen, D., Weniger, T., Supply, P., and Niemann, S. (2008). Evaluation and strategy for use of MIRU-VNTRplus, a multifunctional database for online analysis of genotyping data and phylogenetic identification of Mycobacterium tuberculosis complex isolates. J. Clin. Microbiol. 46, 2692-2699. doi: 10.1128/JCM.00540-08

Bandyopadhyay, B., Das Gupta, T., Roy, D., and Das Gupta, S. K. (2012). DnaK dependence of the mycobacterial stress-responsive regulator HspR is mediated through its hydrophobic C-terminal tail. J. Bacteriol. 194, 4688-4697. doi: 10.1128/JB.00415-12

Bucca, G., Brassington, A. M., Schonfeld, H. J., and Smith, C. P. (2000). The HspR regulon of Streptomyces coelicolor: a role for the DnaK chaperone as a transcriptional co-repressordagger. Mol. Microbiol. 38, 1093-1103. doi: 10.1046/j.1365-2958.2000.02194.x

Champion, P. A., Champion, M. M., Manzanillo, P., and Cox, J. S. (2009). ESX1 secreted virulence factors are recognized by multiple cytosolic AAA ATPases in pathogenic mycobacteria. Mol. Microbiol. 73, 950-962. doi: 10.1111/j.13652958.2009.06821.x

Cole, S., Brosch, R., Parkhill, J., Garnier, T., Churcher, C., Harris, D., et al. (1998). Deciphering the biology of Mycobacterium tuberculosis from the complete genome sequence. Nature 393, 537-544. doi: 10.1038/31159

Cowan, L. S., Hooks, D. P., Christianson, S., Sharma, M. K., Alexander, D. C., Guthrie, J. L., et al. (2012). Evaluation of mycobacterial interspersed repetitiveunit-variable-number tandem-repeat genotyping as performed in laboratories in Canada, France, and the United States. J. Clin. Microbiol. 50, 1830-1831. doi: 10.1128/JCM.00168-12

Da Silva, P. E., Von Groll, A., Martin, A., and Palomino, J. C. (2011). Efflux as a mechanism for drug resistance in Mycobacterium tuberculosis. FEMS Immunol. Med. Microbiol. 63, 1-9. doi: 10.1111/j.1574-695X.2011.00831.x

Dubnau, E., Chan, J., Raynaud, C., Mohan, V. P., Laneelle, M. A., Yu, K., et al. (2000). Oxygenated mycolic acids are necessary for virulence of Mycobacterium tuberculosis in mice. Mol. Microbiol. 36, 630-637. doi: 10.1046/j.13652958.2000.01882.x

Gler, M. T., Skripconoka, V., Sanchez-Garavito, E., Xiao, H., Cabrera-Rivero, J. L., Vargas-Vasquez, D. E., et al. (2012). Delamanid for multidrugresistant pulmonary tuberculosis. N. Engl. J. Med. 366, 2151-2160. doi: 10.1056/NEJMoa1112433

Hartkoorn, R. C., Chandler, B., Owen, A., Ward, S. A., Bertel Squire, S., Back, D. J., et al. (2007). Differential drug susceptibility of intracellular and extracellular tuberculosis, and the impact of P-glycoprotein. Tuberculosis (Edinb.) 87, 248-255. doi: 10.1016/j.tube.2006.12.001

Lalor, M. K., Greig, J., Allamuratova, S., Althomsons, S., Tigay, Z., Khaemraev, A., et al. (2013). Risk factors associated with default from multi- and extensively drug-resistant tuberculosis treatment, Uzbekistan: a retrospective cohort analysis. PLOS ONE 8:e78364. doi: 10.1371/journal.pone.00 78364

Li, S. P., Feng, J. J., Wang, H. G., Wang, X. F., and Lv, Z. J. (2012). The effects of SV40 PolyA sequence and its AATAAA signal on upstream GFP gene expression and transcription termination. Yi Chuan 34, 113-119. doi: 10.3724/SP.J.1005.2012.00113

Liu, B. B., Lu, L. P., Lu, B., Wan, K. L., and Yan, Y. (2012). Meta analysis on the correlation between Mycobacterium tuberculosis Beijing family strains and drug resistance. Zhonghua Yu Fang Yi Xue Za Zhi 46, 158-164. 
Narberhaus, F. (1999). Negative regulation of bacterial heat shock genes. Mol. Microbiol. 31, 1-8. doi: 10.1046/j.1365-2958.1999.01166.x

Nebenzahl-Guimaraes, H., Jacobson, K. R., Farhat, M. R., and Murray, M. B. (2014). Systematic review of allelic exchange experiments aimed at identifying mutations that confer drug resistance in Mycobacterium tuberculosis. J. Antimicrob. Chemother. 69, 331-342. doi: 10.1093/jacl dkt358

Olsen, I., Balasingham, S. V., Davidsen, T., Debebe, E., Rodland, E. A., Van Soolingen, D., et al. (2009). Characterization of the major formamidopyrimidine-DNA glycosylase homolog in Mycobacterium tuberculosis and its linkage to variable tandem repeats. FEMS Immunol. Med. Microbiol. 56, 151-161. doi: 10.1111/j.1574-695X.2009.00562.x

Perez-Lago, L., Navarro, Y., Herranz, M., Bouza, E., and Garcia-De-Viedma, D. (2013). Differences in gene expression between clonal variants of Mycobacterium tuberculosis emerging as a result of microevolution. Int. J. Med. Microbiol. 303, 674-677. doi: 10.1016/j.ijmm.2013.09.010

Pin-Fei, F. (2010). Research on the Resistance Mechanism of Anti-Tuberculous Drugs Based on the Interactions with the Transporter P-gp (in Chinese). Guang Zhou: Zhongshan University press.

Rakash, J., Velpandian, T., Pande, J. N., and Gupta, S. K. (2003). Serum rifampicin levels in patients with tuberculosis: effect of P-Glycoprotein and CYP3A4 blockers on its absorption. Clin. Drug Investig. 23, 463-472. doi: 10.2165/00044011200323070-00005

Refaya, A. K., Sivakumar, S., Sundararaman, B., and Narayanan, S. (2012). Polymorphism in the RD1 locus and its effect on downstream genes among South Indian clinical isolates of Mycobacterium tuberculosis. J. Med. Microbiol. 61, 1352-1359. doi: 10.1099/jmm.0.044453-0

Strong, M., Graeber, T. G., Beeby, M., Pellegrini, M., Thompson, M. J., Yeates, T. O., et al. (2003). Visualization and interpretation of protein networks in Mycobacterium tuberculosis based on hierarchical clustering of genome-wide functional linkage maps. Nucleic Acids Res. 31, 7099-7109. doi: 10.1093/nar/gkg924
Supply, P., Magdalena, J., Himpens, S., and Locht, C. (1997). Identification of novel intergenic repetitive units in a mycobacterial two-component system operon. Mol. Microbiol. 26, 991-1003. doi: 10.1046/j.1365-2958.1997.6361999.x

Supply, P., Mazars, E., Lesjean, S., Vincent, V., Gicquel, B., and Locht, C. (2000). Variable human minisatellite-like regions in the Mycobacterium tuberculosis genome. Mol. Microbiol. 36, 762-771. doi: 10.1046/j.1365-2958.2000.01905.x

Tantivitayakul, P., Panapruksachat, S., Billamas, P., and Palittapongarnpim, P. (2010). Variable number of tandem repeat sequences act as regulatory elements in Mycobacterium tuberculosis. Tuberculosis (Edinb.) 90, 311-318. doi: 10.1016/j.tube.2010.08.003

Xin, Y., Yin, F., Qi, S., Shen, L., Xu, Y., Luo, L., et al. (2013). Parthenolide reverses doxorubicin resistance in human lung carcinoma A549 cells by attenuating NF-kappaB activation and HSP70 up-regulation. Toxicol. Lett. 221, 73-82. doi: 10.1016/j.toxlet.2013.06.215

Conflict of Interest Statement: The authors declare that the research was conducted in the absence of any commercial or financial relationships that could be construed as a potential conflict of interest.

Received: 28 November 2014; accepted: 09 February 2015; published online: 24 February 2015.

Citation: Yu-feng W, Chao J and Xian-feng C (2015) Drug-resistant tuberculosis can be predicted by Mycobacterial interspersed repetitive unit locus. Front. Microbiol. 6:147. doi: 10.3389/fmicb.2015.00147

This article was submitted to Antimicrobials, Resistance and Chemotherapy, a section of the journal Frontiers in Microbiology.

Copyright (๑) $2015 \mathrm{Yu}$-feng, Chao and Xian-feng. This is an open-access article distributed under the terms of the Creative Commons Attribution License (CC BY). The use, distribution or reproduction in other forums is permitted, provided the original author(s) or licensor are credited and that the original publication in this journal is cited, in accordance with accepted academic practice. No use, distribution or reproduction is permitted which does not comply with these terms. 